\title{
GROUP-QUOTIENTS WITH POSITIVE SECTIONAL CURVATURES ${ }^{1}$
}

\author{
ROBERT GEROCH
}

\begin{abstract}
Let $H$ be a closed subgroup of compact Lie group $G$. A necessary and sufficient condition is obtained for the existence of a leftinvariant Riemannian metric on $G$ such that the subduced metric on the quotient $H \backslash G$ has strictly positive sectional curvatures.
\end{abstract}

Which compact manifolds admit a Riemannian metric with positive sectional curvatures? Although it is known that a few manifolds (e.g., among others, the spheres) do, and that a few (e.g., those which are orientable, even-dimensional, and not simply connected [4]) do not, this question is largely open. Since the direct search for additional examples is hampered by the computational difficulties in obtaining explicitly the curvature tensors of various trial metrics, one looks for situations in which these computations are simplified. One such situation, for example, is that of the homogeneous Riemannian manifolds, for which the determination of the sectional curvatures reduces to an algebraic problem in the Lie algebra of a certain group. All compact homogeneous Riemannian manifolds with positive sectional curvatures- of which there turn out to be suprisingly few-have been found [5]. There is, however, a much larger class of potential examples which shares with the homogeneous case the advantage of a purely algebraic formulation.

The construction with which we shall be concerned is the following. Let $G$ be a compact Lie group, $H$ a closed subgroup, and let $\pi: G \rightarrow H \backslash G$ be the projection of $G$ onto the manifold $M$ of right cosets of $H$ in $G$. Then, for $\gamma$ any left-invariant metric on $G, \pi$ is a Riemannian submersion to a certain (the subduced) metric on $M$. We shall obtain a simple necessary and sufficient condition for the existence of such a $\gamma$ for which the Riemannian manifold $M$ has positive sectional curvatures.

We remark that $M$ need not be homogeneous. Indeed, in many cases (typically, for $H$ sufficiently large and $\gamma$ sufficiently general) the isometry group of $M$ is the trivial group. Of course, were $\gamma$ instead right-invariant, or $M$ instead the left cosets of $H$ in $G$, then $\pi$ would in general not be a Riemannian submersion to any metric on $M$. Numerous other constructions

Received by the editors December 30, 1976.

AMS (MOS) subject classifications (1970). Primary 53B20.

${ }^{1}$ Supported in part by the National Science Foundation, under contract number MPS 74 17456. 
involving groups turn out to be special cases of this one. For example, let $K$ be a compact Lie group, and let $\psi: K \times K \rightarrow K$ have action $\psi(x, y)=x y$. On $K \times K$ impose Riemannian metric $\gamma_{r} \times \gamma_{l}$, where $\gamma_{r}$ and $\gamma_{l}$ are right- and left-invariant metrics on $K$, respectively. Then-but in general only for these left-right choices $-\psi$ is a Riemannian submersion to a metric on $K$. This is a special case of the present construction, with $G=K^{-1} \times K$ and $H$ the diagonal subgroup.

Denote by $g$ and $\mathfrak{h}$ the Lie algebras of $G$ and $H$, respectively. Fix once and for all a bi-invariant metric $p$ on $g$, i.e., a metric satisfying

$$
p(X,[Y, Z])=-p(Y,[X, Z])
$$

for all $X, Y, Z \in \mathrm{g}$. [For example, in the semisimple case $p$ can be minus the Killing form.] Nothing of what follows depends substantially on this choice of p. Our main result is

TheOREM. Let $G \rightarrow{ }^{\pi} H \backslash G=M$, Lie algebras $\mathfrak{g}$ and $\mathfrak{h}$, and bi-invariant metric $p$ be as above. Then there exists a left-invariant Riemannian metric on $G$ such that the subduced metric on $M$ has positive sectional curvatures if and only if no two independent vectors in $\mathfrak{g}$, p-orthogonal to $\mathfrak{h}$, commute.

We illustrate the application of this Theorem with three examples.

For the first, set $H=\{e\}$. Then $\mathfrak{h}=\{0\}$, every vector in $\mathfrak{g}$ is $p$-orthogonal to $\mathfrak{h}$, and $M=G$. The Theorem then states that $G$ itself admits a leftinvariant metric with positive sectional curvatures if and only if no two independent vectors in $g$ commute, i.e., if and only if $G$ has rank not exceeding one, i.e., (now ignoring the trivial cases with $\operatorname{dim} G \leqslant 1$ ) if and only if the universal covering group of the connected component of the identity of $G$ is the Lie group $S U(2)$. This result is known [5].

No example is known of two compact, nonzero dimensional manifolds whose product admits a metric of positive sectional curvatures. Consider the following class of potential examples. Let $H$ and $H^{\prime}$ be closed subgroups of compact Lie groups $G$ and $G^{\prime}$, respectively. Then $(H \backslash G) \times\left(H^{\prime} \backslash G^{\prime}\right) \approx(H$ $\left.\times H^{\prime}\right) \backslash\left(G \times G^{\prime}\right)=M$ is a product manifold. Try to find a left-invariant metric on $G \times G^{\prime}$, not necessarily a product metric, such that the subduced metric on $M$ has positive sectional curvatures. It follows from the Theorem that all examples so constructed must fail: Choose nonzero vector in the Lie algebra of $G$ (resp., of $G^{\prime}$ ) p-orthogonal to the Lie algebra of $H$ (resp., of $H^{\prime}$ ). Then these two vectors, regarded as elements of the Lie algebra of $G \times G^{\prime}$, commute.

The third application is to the Hopf conjecture: A compact, even-dimensional Riemannian manifold with positive sectional curvatures has positive Euler number. We have

COROLlary. The Hopf conjecture is true for any Riemannian manifold $M$ constructed as in the Theorem.

Proof. Let $M$ be even-dimensional, and have nonpositive Euler number. 
Then even-dimensionality implies that $H$ has even co-rank in $G$ (for a Lie group is even-dimensional if and only if its rank is even), while nonpositivity of the Euler number implies that $H$ has positive co-rank in $G$ [3]. Hence, the co-rank of $H$ in $G$ must be greater than or equal to two. Let $c_{\mathfrak{b}}$ be a Cartan subalgebra of $\mathfrak{h}$, and extend $\mathfrak{c}_{\mathfrak{h}}$ to a Cartan subalgebra, $\mathfrak{c}_{\mathfrak{g}}$, of $\mathfrak{g}$. Let, by the above, $X$ and $Y$ be independent elements of $c_{\mathfrak{g}}$ which are $p$-orthogonal to $c_{\mathfrak{h}}$. Then in particular $[X, Y]=0$. But every element of $\mathfrak{h}$ can be written in the form $U+[Z, V]$ with $U, V \in \mathfrak{c}_{\mathfrak{h}}$ and $Z \in \mathfrak{h}$, while $p(X, U+[Z, V])=$ $p(X, U)-p(Z,[X, V])=0$. Thus, $X$, and similarly $Y$, is $p$-orthogonal to $\mathfrak{h}$. That the subduced metric on $M$ cannot have positive sectional curvatures now follows from the Theorem.

The proof of the Theorem requires some notation and a preliminary formula. For $\gamma$ a left-invariant metric on $G$, set $g=\left.\gamma\right|_{e}$, so $g$, a metric on the vector space $g$, uniquely determines $\gamma$. Define invertible $\hat{g}: \mathfrak{g} \rightarrow \mathfrak{g}$ by $g(\hat{g} X, Y)$ $=p(X, Y)$, and metric $\bar{g}$ on $g$ by $\bar{g}(X, Y)=g(\hat{g} X, \hat{g} Y)$, for all $X, Y \in g$. For any $x \in G$, denote by $\mathcal{L}_{x}$ (resp., $\Re_{x}$ ) the diffeomorphism on $G$ given by left-multiplication (resp., right-multiplication) by $x$. Denote $\left(\varrho_{x^{-1}} \circ \Re_{x}\right)_{*}$, restricted to $\mathrm{g}$, by $\mathrm{Aut}_{x}$, so $\mathrm{Aut}_{x}$ is an automorphism on the Lie algebra $\mathrm{g}$, and a $p$-isometry. Let $x \in G$. We define an isomorphism, $g_{x}$, from the tangent space of $M$ at the point $\pi(x)$ to the subspace of $g$ consisting of elements $p$-orthogonal to Aut ${ }_{x}[\mathfrak{b}]$, as follows. For $\alpha$ tangent to $M$ at $\pi(x)$, let $\zeta$ be the unique tangent vector of $G$ at $x$ such that $\pi_{*}(\zeta)=\alpha$, and such that $\zeta$ is $\gamma$-orthogonal to $\Re_{x^{*}}[\mathfrak{h}]$, the space of tangents to the $H$-coset through $x$. Then, by left-invariance of $\gamma$, the element $\mathcal{L}_{x^{-1} *}(\zeta)$ of $g$ is $g$-orthogonal to the subspace $\mathscr{L}_{x^{-1} *}\left[\Re_{x *}[\mathfrak{l}]\right]=$ Aut $_{x}[\mathfrak{h}]$ of $\mathfrak{g}$. Hence, $\hat{g}^{-1}\left(\mathscr{L}_{x^{-1}}(\zeta)\right)$, the desired $g_{x}(\alpha)$, is $p$-orthogonal to Aut ${ }_{x}[\mathfrak{h}]$. A straightforward calculation (see Appendix) using O'Neill's formula [2] yields: For $\alpha$ and $\beta$ tangents of $M$ at $\pi(x)$, the $(\alpha, \beta)$-sectional curvature of $M$ is given, with $X=g_{x}(\alpha), Y=g_{x}(\beta)$, by the right side of

$R(X, Y)=-\frac{3}{4} g([\hat{g} X, \hat{g} Y],[\hat{g} X, \hat{g} Y])$

$$
\begin{aligned}
& +\frac{1}{2} p([\hat{g} X, \hat{g} Y],[X, \hat{g} Y]+[\hat{g} X, Y])-\bar{g}([X, \hat{g} X],[Y, \hat{g} Y]) \\
& +\frac{1}{4} \bar{g}([X, \hat{g} Y]-[\hat{g} X, Y],[X, \hat{g} Y]-[\hat{g} X, Y])+\frac{3}{4} g(T, T)
\end{aligned}
$$

where

$$
T=\operatorname{proj}([\hat{g} X, \hat{g} Y]-\hat{g}[\hat{g} X, Y]-\hat{g}[X, \hat{g} Y]),
$$

and where proj: $\mathfrak{g} \rightarrow \mathfrak{g}$ takes the $g$-projection into the subspace $\mathrm{Aut}_{x}[\mathfrak{h}]$ of $\mathfrak{g}$. Thus,

LEMMA. The quotient $M$ has positive sectional curvatures at $\pi(x)$ if and only if, for all independent $X, Y \in \mathfrak{g}$,p-orthogonal to $\mathrm{Aut}_{x}[\mathfrak{h}], R(X, Y)>0$.

Proof of THE Theorem. For the "if" part, let no two independent vectors in $\mathfrak{g}, p$-orthogonal to $\mathfrak{h}$, commute. Then certainly no two independent vectors in $g, p$-orthogonal to Aut $\left[\mathfrak{h}_{x}\right]$ for any $x$, can commute. Set $g=p$, so $\hat{g}$ is the 
identity and $\bar{g}=p$. Making these substitutions in (2), the third and fourth terms on the right give zero while the last term on the right is nonnegative, and so we obtain $R(X, Y) \geqslant \frac{1}{4} p([X, Y],[X, Y])$. The result is immediate from the Lemma.

For the converse, let $X^{\prime}$ be any generic element of $g$, i.e., such that the subspace of $g$ consisting of elements which commute with $X^{\prime}$ has minimal dimension, and let $Y^{\prime}$ commute with $X^{\prime}$. Set, for $x \in G, f(x)=\bar{g}\left(\operatorname{Aut}_{x}\left(X^{\prime}\right)\right.$, Aut $_{x}\left(X^{\prime}\right)$ ), so $f$ is a smooth function on $G$. Let $x=x_{0}$ maximize $f$, and set $X_{0}=\operatorname{Aut}_{x_{0}}\left(X^{\prime}\right), Y_{0}=\operatorname{Aut}_{x_{0}}\left(Y^{\prime}\right)$, so $X_{0}$ is also generic, and also $\left[X_{0}, Y_{0}\right]=0$. Then $\bar{g}\left(\operatorname{Aut}_{x}\left(X_{0}\right), \operatorname{Aut}_{x}\left(X_{0}\right)\right)$ is maximal at $x=e$. Equating the first $x$ derivative of this function, evaluated at $x=e$, to zero, we obtain $\bar{g}\left(X_{0}\right.$, $\left.\left[X_{0}, Z\right]\right)=0$ for every $Z \in \mathrm{g}$. But using (1) and that $Z$ is arbitrary, this is equivalent to

$$
\left[X_{0}, \hat{g} X_{0}\right]=0 .
$$

The statement that the second $x$-derivative of $\bar{g}\left(\operatorname{Aut}_{x}\left(X_{0}\right), \operatorname{Aut}_{x}\left(X_{0}\right)\right)$ be nonpositive-definite at $x=e$ is: $\bar{g}\left(\left[X_{0}, Z\right],\left[X_{0}, Z\right]\right)+\bar{g}\left(X_{0},\left[\left[X_{0}, Z\right], Z\right]\right) \leqslant 0$ for every $Z \in \mathrm{g}$. Using (1), this may be rewritten

$$
\bar{g}\left(\left[X_{0}, Z\right],\left[X_{0}, Z\right]\right)-p\left(\left[\hat{g} X_{0}, Z\right],\left[X_{0}, Z\right]\right)<0 .
$$

Equation (5) implies in particular: Every element of $\mathfrak{g}$ which commutes with $\hat{g} X_{0}$ also commutes with $X_{0}$. But $X_{0}$ is generic, and so the converse also holds. In particular, since $\left[X_{0}, Y_{0}\right]=0$, we have $\left[\hat{g} X_{0}, Y_{0}\right]=0$. Substituting this and (4) into Equation (2), using for the last term the fact that the norm of the projection of a vector cannot exceed the norm of that vector, we obtain

$$
\begin{aligned}
R\left(X_{0}, Y_{0}\right) \leqslant & -p\left(\left[\hat{g} X_{0}, \hat{g} Y_{0}\right],\left[X_{0}, \hat{g} Y_{0}\right]\right) \\
& +\bar{g}\left(\left[X_{0}, \hat{g} Y_{0}\right],\left[X_{0}, \hat{g} Y_{0}\right]\right) .
\end{aligned}
$$

But the right side of (6) is nonpositive, by (5) with $Z=\hat{g} Y_{0}$. We conclude: For $X^{\prime}, Y^{\prime} \in \mathrm{g}$, with $X^{\prime}$ generic and $\left[X^{\prime}, Y^{\prime}\right]=0$, there exists $x_{0} \in G$ such that

$$
R\left(\operatorname{Aut}_{x_{0}}\left(X^{\prime}\right), \operatorname{Aut}_{x_{0}}\left(Y^{\prime}\right)\right) \leqslant 0 .
$$

Now let $X, Y \in \mathfrak{g}$, with $[X, Y]=0$, be $p$-orthogonal to $\mathfrak{h}$. Choose sequences $X_{i}, Y_{i} \in \mathfrak{g}(i=1,2, \ldots)$ such that the $X_{i}$ approach $X$ through generic elements, the $Y_{i}$ approach $Y$, and $\left[X_{i}, Y_{i}\right]=0$ for all $i$. By the conclusion above, there exists a sequence $x_{i} \in G$ with

$$
R\left(\operatorname{Aut}_{x_{i}}\left(X_{i}\right), \operatorname{Aut}_{x_{i}}\left(Y_{i}\right)\right) \leqslant 0
$$

for every $i$. By compactness of $G$, the $x_{i}$ accumulate at some $x \in G$; by continuity, $R\left(\operatorname{Aut}_{x}(X), \operatorname{Aut}_{x}(Y)\right) \leqslant 0$. But $\operatorname{Aut}_{x}(X)$ and $\operatorname{Aut}_{x}(Y)$ are $p$ orthogonal to $A_{x}[\mathrm{~h}]$. By the Lemma, there is a zero of the sectional curvatures of $M$, at the point $\pi(x)$.

We note, from the first paragraph of this proof, that references [1] and [5] provide a classification of all quotients, $H \backslash G$, which admit such a subduced 
metric with positive sectional curvatures.

Appendix. We sketch the calculation which yields that, for $\alpha$ and $\beta$ tangents of $M$ at $\pi(x)$, the $(\alpha, \beta)$-sectional curvature of $M$ is $R\left(G_{x}(\alpha), g_{x}(\beta)\right)$, given by equation (2).

Denote sectional curvatures of $G$ and $M$ by $R_{G}\left(\right.$, ) and $R_{M}($, ), respectively. Let $\alpha, \beta$ be tangents of $M$ at $\pi(x)$, and set $X=g_{x}(\alpha)$ and $Y=g_{x}(\beta)$. By definition of $g_{x}, \mathcal{L}_{x *}(\hat{g} X)$ and $\mathcal{L}_{x *}(\hat{g} Y)$ are the lifts of $\alpha, \beta$ to tangent vectors at $x \gamma$-orthogonal to $\mathcal{R}_{x *}[\mathfrak{l}]$ ]. By O’Neill's formula [2], we have the first equality of

$$
\begin{aligned}
R_{M}(\alpha, \beta) & =R_{G}\left(\mathscr{E}_{x *}(\hat{g} X), \mathscr{L}_{x *}(\hat{g} Y)\right)+\frac{3}{4} \gamma\left(T^{\prime \prime}, T^{\prime \prime}\right) \\
& =R_{G}(\hat{g} X, \hat{g} Y)+\frac{3}{4} g\left(T^{\prime}, T^{\prime}\right)
\end{aligned}
$$

where $T^{\prime \prime}$ is the $\gamma$-projection into $\Re_{x *}[\mathfrak{h}]$ at $x$ of the Lie bracket (as vector fields) of any extensions of $\mathcal{E}_{x *}(\hat{g} X)$ and $\mathcal{E}_{x *}(\hat{g} Y)$ to vector fields defined in a neighborhood of $x$ and everywhere $\gamma$-orthogonal to the $\Re_{y *}[\mathfrak{h}]$. The second equality follows from left-invariance of $\gamma$, where $T^{\prime}=\mathcal{E}_{x^{-1} *}\left(T^{\prime \prime}\right)$ is the $g$-projection into Aut $_{x}[\mathfrak{h}]$ at $e$ of the Lie bracket of any extensions of $\hat{g} X$ and $\hat{g} Y$ to vector fields defined in a neighborhood of $e$ and everywhere $\gamma$ orthogonal to the $\Re_{y *}\left[\mathrm{Aut}_{x}[\mathfrak{h}]\right]$. We shall show that (i) $R_{G}(\hat{g} X, \hat{g} Y)$ is given by the sum of the first four terms on the right in (2), and (ii) $T^{\prime}$ is given by the $T$ of equation (3).

(i) Denote by $\psi$ the mapping which assigns to each tensor over $g$ that left-invariant tensor field on $G$ whose value at $e$ is the given tensor. Denote by $\nabla$ the torsion-free derivative operator on $G$ compatible with the left-invariant metric $\gamma=\psi(g)$. Then, for any $U, V \in g$, the right side of

$$
\psi(\Gamma(U, V))=\nabla_{\psi(U)} \psi(V)
$$

is left-invariant, whence there exists bilinear $\Gamma: g \times g \rightarrow g$ such that (8) holds for all $U, V$. Since $\nabla$ is torsion-free, and annihilates the metric $\psi(g)$, we have

$$
\begin{gathered}
\Gamma(U, V)-\Gamma(V, U)=[U, V], \\
g(\Gamma(W, U), V)+g(\Gamma(W, V), U)=0,
\end{gathered}
$$

respectively, for all $U, V, W \in g$. These two equations determine $\Gamma$ uniquely. Since the $\Gamma$ 's defined by demanding either

$$
\begin{aligned}
g(W, \Gamma(U, V))= & \frac{1}{2} p\left(V,\left[\hat{g}^{-1} W, U\right]+\left[\hat{g}^{-1} U, W\right]\right) \\
& +\frac{1}{2} g(V,[W, U])
\end{aligned}
$$

or

$$
\begin{aligned}
p(W, \Gamma(U, V))= & -\frac{1}{2} g\left(W,\left[\hat{g}^{-1} U, V\right]+\left[\hat{g}^{-1} V, U\right]\right) \\
& +\frac{1}{2} p(W,[U, V])
\end{aligned}
$$

for all $U, V, W \in g$ satisfy both (9) and (10), Equations (11) and (12) are true. 
Denoting by $\bar{\Re}$ the curvature tensor of $G, \psi(g)$, we have

$$
\begin{aligned}
\bar{R}(\psi(U), \psi(V))(\psi(V))= & \nabla_{\psi(U)} \nabla_{\psi(V) \psi(V)} \\
& -\nabla_{\psi(V)} \nabla_{\psi(U)} \psi(V)-\nabla_{[\psi(U), \psi(V)] \psi(V) .}
\end{aligned}
$$

Evaluating (13) at the identity using (8), and then taking the $g$-inner product with $U$, we obtain for the $(U, V)$-sectional curvature of $G$

$$
\begin{aligned}
R_{G}(U, V)= & g(U, \Gamma(U, \Gamma(V, V)))-g(U, \Gamma(V, \Gamma(U, V))) \\
& -g(U, \Gamma([U, V], V)) .
\end{aligned}
$$

Now eliminate the first " $\Gamma$ " in each term on the right in (14) using (11), then the remaining " $\Gamma$ 's" using (12), and set $U=\hat{g} X, V=\hat{g} Y$.

(ii) Let $U, V \in g$; set $\mu=\psi(U), \nu=\psi(V)$. Let $\sigma$ and $\tau$ be vector fields defined in a neighborhood of $e$ which vanish at $e$ and which are such that $\mu+\sigma$ and $\nu+\tau$ are $\gamma$-orthogonal to the $R_{y *}\left[\right.$ Aut $\left._{x}[\mathfrak{k}]\right]$. Then $T^{\prime}$ is the $g$-projection into Aut $x_{x}[\mathfrak{h}]$ of $\underline{\varrho}_{\mu+\sigma}(\nu+\tau)$, where $\underline{\underline{Q}}$ denotes the Lie derivative. Let $\kappa$ be any right-invariant vector field in the $\Re_{y *}\left[\right.$ Aut $\left._{x}[\mathfrak{h}]\right]$, and set $K=\left.\kappa\right|_{e}$. Then

$$
\begin{aligned}
g\left(K, T^{\prime}\right) & =\left.\gamma\left(\kappa, \underline{\varrho}_{\mu+\sigma}(\nu+\tau)\right)\right|_{e} \\
& =\left.\gamma\left(\kappa, \underline{\varrho}_{\mu} \nu\right)\right|_{e}+\left.\gamma\left(\kappa, \underline{\varrho}_{\mu} \tau\right)\right|_{e}+\gamma\left(\kappa, \underline{\varrho}_{\sigma \nu}\right)\left|+\gamma\left(\kappa, \underline{\varrho}_{\sigma} \tau\right)\right|_{e} .
\end{aligned}
$$

The first term on the right is $g(K,[U, V])$; the fourth zero. For the second term on the right, we have $\left.\gamma\left(\kappa, \underline{\varrho}_{\mu} \tau\right)\right|_{e}=\left.\underline{\varrho}_{\mu} \gamma(\kappa, \tau)\right|_{e}=-\left.\underline{\varrho}_{\mu} \gamma(\kappa, \nu)\right|_{e}=$ $-\left.\underline{\mathcal{E}}_{\mu} \psi(p)\left(\kappa, \psi\left(\hat{\mathrm{g}}^{-1}\right) \nu\right)\right|_{e}=-\left.\psi(p)\left(\kappa, \underline{\mathcal{E}}_{\mu} \psi\left(\hat{\mathrm{g}}^{-1}\right) \nu\right)\right|_{e}=-p\left(K,\left[U, \hat{g}^{-1} V\right]\right)=$ $-g\left(K, \hat{g}\left[U, \hat{g}^{-1} V\right]\right)$. [We used the vanishing of $\tau$ at $e$ in the first step; that $\gamma(\kappa, \nu+\tau)=0$ in the second; and $\underline{\varrho}_{\mu} \psi(p)=0$ and $\underline{\varrho}_{\mu} \kappa=0$ in the fourth.] Similarly for the third term on the right in (15). Making these substitutions in (15), we obtain

$$
\begin{aligned}
g\left(K, T^{\prime}\right) & =g(K,[U, V])-g\left(K, \hat{g}\left[U, \hat{g}^{-1} V\right]\right)-g\left(K, \hat{g}\left[\hat{g}^{-1} U, V\right]\right) \\
& =g(K,[U, V])-\hat{g}\left[U, \hat{g}^{-1} V\right]-\hat{g}\left(\left[\hat{g}^{-1} U, V\right]\right) .
\end{aligned}
$$

Setting $U=\hat{g} X, V=\hat{g} Y$, comparing (16) with (3), and using the fact that $K \in \mathrm{Aut}_{x}[\mathfrak{h}]$ is arbitrary, the result, $T^{\prime}=T$, follows.

\section{REFERENCES}

1. L. Berard Bergery, Sur certaines fibrations d'espaces homogenes Riemanniens, Compositio Math. 30 (1975), 43-61; Les variétés Riemanniennes homogenes simplement connexes de dimension impaire a courbure strictement positive, J. Math. Pures Appl. 55 (1976), 47-68.

2. B. O'Neill, The fundamental equations of a submersion, Michigan Math. J. 13 (1966), 459-469.

3. H. Samelson, On the curvature and characteristic of homogeneous spaces, Michigan Math. J. 5 (1958), 13-18.

4. J. L. Synge, The first and second variations of the length-integral in Riemannian space, Proc. London Math. Soc. (2) 25 (1926), 247-264.

5. N. R. Wallach, Compact homogeneous Riemannian manifolds with strictly positive curvature, Ann. of Math. (2) 96 (1972), 277-295.

Department of Mathematics, University of Chicago, Chicago, Illinois 60637 